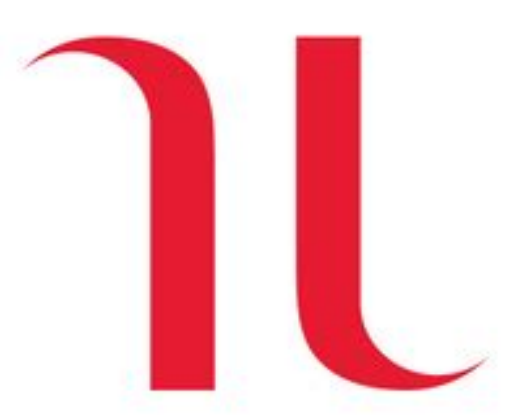

L'exposition de la télévision dans l'entre-deux-guerres

Author(s): Anne-Katrin Weber

Source: Relations internationales, Hiver 2016 (janvier-mars), No. 164, Les expositions internationales, mises en scène de la modernité (Hiver 2016 (janvier-mars)), pp. 75-92

Published by: Editions Belin

Stable URL: https://www.jstor.org/stable/10.2307/48611111

JSTOR is a not-for-profit service that helps scholars, researchers, and students discover, use, and build upon a wide range of content in a trusted digital archive. We use information technology and tools to increase productivity and facilitate new forms of scholarship. For more information about JSTOR, please contact support@jstor.org.

Your use of the JSTOR archive indicates your acceptance of the Terms \& Conditions of Use, available at https://about.jstor.org/terms

This article discusses television's political and symbolic construction in the interwar period in Germany, Great Britain and the United States. It analyzes the public presentations of the new media organized in connection with the launching of public services in Berlin (1935), London (1936) and New York (1939), and examines how exhibitions actively shaped television as a national medium in a context of international competition and transnational exchanges. 


\section{L'exposition de la télévision dans l'entre-deux-guerres : entre appropriations nationales et échanges transnationaux}

Dès le milieu des années 1920 et le développement des premiers prototypes fonctionnels, la télévision se fait objet d'exposition. La nouvelle technologie est montrée dans des grands magasins et des foires industrielles, à l'occasion d'expositions nationales et universelles. Si de nombreux problèmes techniques, économiques et organisationnels empêchent le lancement de services télévisuels nationaux avant les années 1950, ces démonstrations publiques font de la télévision un média qui circule hors des espaces scientifiques et industriels : présentée à une foule curieuse et souvent très enthousiaste, la télévision est vue et regardée avant même qu'elle ne diffuse de programme régulier. Célébrée dans la presse et les discours officiels, cette exposition du média sert fréquemment de témoin de l'avancée technoscientifique d'une nation. Par son caractère nouveau et spectaculaire, la "vision à distance » est censée matérialiser le savoir-faire de l'industrie nationale et placer celle-ci au premier plan sur la scène internationale. À l'instar d'autres technologies contemporaines, elle est intégrée aux mises en scènes ritualisées de l'identité nationale et participe à la promotion des sentiments patriotiques ; elle nourrit l'image d'une "communauté imaginaire " fondée sur le progrès et incarne la modernité d'une nation ${ }^{1}$.

Cependant l'histoire télévisuelle interroge une lecture qui serait fondée sur la seule perspective nationale. D'un côté, la télévision dans l'entre-deuxguerres est un média "ultra-local»: en raison des limitations techniques, le récepteur et l'émetteur sont posés à quelques mètres seulement l'un de l'autre lors des premières démonstrations. Durant les années 1930, la transmission télévisuelle est toujours restreinte à un périmètre de quelques kilomètres, généralement une fraction de la superficie des grandes métropoles (Berlin, Londres, New York). D’un autre côté, la télévision est un média international : marqués par des échanges intenses de savoirs, de personnes et d'objets, les recherches et les développements télévisuels dépendent de

1. Benedict Anderson, Imagined Communities: Reflections on the Origin and Spread of Nationalism, Londres, Verso Editions, 1983.

Relations internationales, $\mathrm{n}^{\circ} 164 / 2016$ 
réseaux scientifiques et industriels transnationaux. La conception nationale de la télévision est finalement remise en cause par sa propriété médiatique. Dès les premières inventions imaginaires et scientifiques de la "vision à distance " à la fin du $\mathrm{XIX}^{\mathrm{e}}$ siècle, la télé-vision promet une ubiquité communicationnelle. Le nœud conceptuel du média est formé par l'utopie de l'abolition de l'espace via la technologie audiovisuelle : celle-ci ne se confine pas aux frontières nationales, mais, au contraire, facilite le rapprochement entre des espaces lointains.

Situé au croisement de l'histoire de la télévision et de celle des expositions, cet article vise à discuter la construction politique et culturelle du média, entre appropriations nationales et échanges transnationaux. À travers l'étude des présentations publiques organisées en lien avec l'inauguration de services publics télévisuels à Berlin, à Londres et à New York dans les années 1930, il examine comment l'exposition de la télévision participe de la définition nationale du média et étudie l'importance du contexte international pour sa "nationalisation ». L'Allemagne, la Grande-Bretagne et les États-Unis, les pays les plus impliqués dans la recherche télévisuelle durant l'entre-deux-guerres, se livrent au milieu de la décennie à une véritable "course à la télévision". Dans ces trois États, la forte valeur symbolique de la télévision comme emblème de progrès scientifique et économique est explicite lors de l'ouverture des premiers services télévisuels réguliers et se traduit dans les salles d'exposition par des scénographies entièrement nouvelles. Malgré des contextes politiques et économiques très différents, l'espace de l'exposition joue un rôle central dans la consolidation des institutions télévisuelles nationales et prépare ou accompagne le lancement du service public télévisuel.

\section{L'IMAGINAIRE TÉLÉVISUEL DE L'UBIQUITÉ COMMUNICATIONNELLE}

Dès la constitution de l'imaginaire télévisuel à la fin du XIX ${ }^{\mathrm{e}}$ siècle, la télévision se caractérise par l'association de la vision à distance et de l'instantanéité communicationnelle : la relation spatio-temporelle du média résumée dans le préfixe "télé- » le rapproche des moyens de communication voisins que sont la télégraphie et la téléphonie. Le nœud conceptuel de la télévision est formé par l'utopie de l'abolition de l'espace via une technologie audiovisuelle. Ainsi, le "téléphonoscope » de George du Maurier, publié en 1878 dans le magazine satirique Punch, établit-il la communication entre des parents qui se trouvent à Londres et leurs enfants installés à Ceylan. Effaçant la distance physique, le téléphonoscope facilite le partage de moments récréatifs et permet aux parents de garder un œil sur leur progéniture. Plus généralement, grâce au visiophone, la présence physique des enfants aux colonies est reproduite et intégrée dans une chambre londonienne (Fig. 1). 


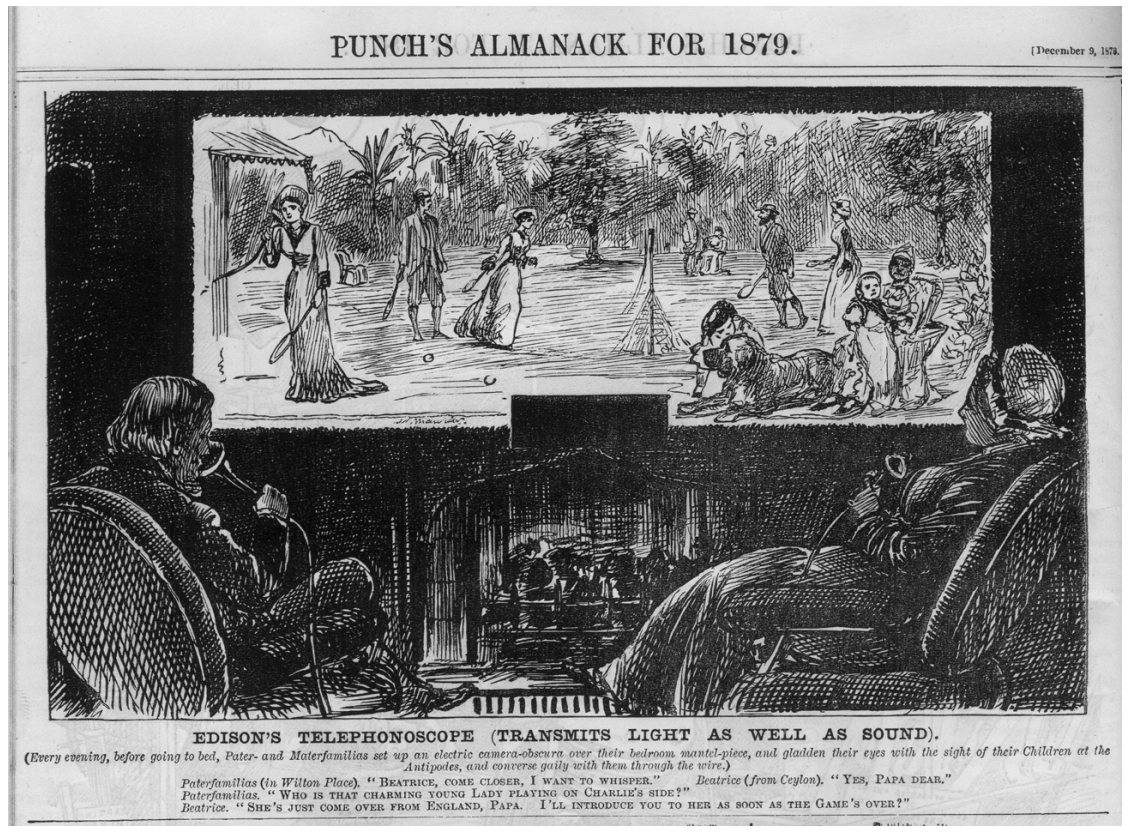

Fig. 1 : George du Maurier, «Edison's Telephonoscope. Transmits Light as well as Sound " ( Le téléphonoscope d'Edison. Transmets la lumière aussi bien que le son »)2, Punch's Almanack for 1879, 9 décembre 1878.

Dans son roman futuriste Le Vingtième Siècle (1883), Albert Robida accorde une place centrale à un appareil similaire et homonyme, à la fois média de divertissement, d'information et de surveillance ${ }^{3}$. Les fonctions du téléphonoscope chez Robida dépendent pareillement de la connectivité instantanée articulée à son pouvoir ubiquitaire. Ainsi, façonnés avant l'émergence des premiers systèmes télévisuels fonctionnels, ces dispositifs imaginaires sont-ils nourris par les promesses des nouveaux moyens de

2. Dialogue sous la légende : Every evening, before going to bed, Pater- and Materfamilias set up an electric camera-obscura over their bedroom mantel-piece, and gladden their eyes with the sight of their children at the Antipodes, and converse gaily with them through the wire.).

Paterfamilias (in Wilton Place): "Beatrice, come closer, I want to whisper".

Beatrice (from Ceylon): "Yes, Papa dear".

Paterfamilias: "Who is that charming young lady playing on Charlie's side? "

Beatrice: "She's just come over from England, Papa. I'll introduce you to her as soon as the game's over?"

(Chaque soir, avant d'aller au lit, Pater- et Materfamilias allument une chambre obscure électrique sur la cheminée de leur chambre à coucher, se réjouissent de voir leurs enfants aux antipodes et conversent gaiment avec eux grâce au fil :

Paterfamilias (à Wilton Place) : «Béatrice, vient plus près, j’ai besoin de te parler discrètement. " Béatrice (depuis Ceylan) : "Oui, mon petit papa. "

Paterfamilias : "Qui est cette jeune et charmante dame qui joue aux côtés de Charlie ?"

Béatrice : "Elle vient juste d'arriver d'Angleterre, Papa. Je te présenterai dès la fin du jeu »).

3. Albert Robida, Le Vingtième Siècle, Paris, G. Decaux, 1883. 
communication et de transport et matérialisent-ils une nouvelle organisation mondiale caractérisée par "l'abolition de l'espace par le temps ${ }^{4}$ ".

Durant l'entre-deux-guerres, le théoricien d'art Rudolf Arnheim propose une utopie davantage universaliste, qui souligne le potentiel de la télévision pour relier les peuples :

Nous voyons les gens rassemblés sur la place centrale d'une cité proche, nous voyons le chef du gouvernement d'un État voisin, nous voyons des boxeurs combattant pour le titre mondial de l'autre côté de l'océan, nous voyons un orchestre de jazz britannique, une soprano italienne, un professeur allemand, les wagons en flamme d'un train en collision, les personnages masqués d'un carnaval [...] nous pouvons admirer le soleil se couchant derrière le Vésuve et, une seconde plus tard, les éclairages nocturnes de New York. Le besoin de la description disparaît en même temps que s'évanouit la barrière de la langue étrangère. Le monde dans toute sa vastitude entre chez nous ${ }^{5}$.

Pour Arnheim, les qualités communicationnelles du média engendrent une cartographie d'États-nations apparaissant simultanément - de manière non hiérarchique - sur l'écran. La vision à distance recrée l'espace public dans l'intimité du privé et produit une nouvelle topographie d'un monde devenu instantanément accessible.

\section{LA «VISION À DISTANCE », DE PRÈS}

Cette conception universaliste de la télévision se heurte néanmoins à de nombreux problèmes techniques, politiques et organisationnels qui empêchent la mise en place d'un réseau télévisuel suffisamment développé pour concrétiser les différents rêves de la « vision à distance ". Même après l'ouverture d'un service public à Berlin en 1935, à Londres en 1936, et à New York en 1939, seul un public restreint (et privilégié) a accès aux programmes : à New York, quelque 800 ménages possèdent un récepteur ; en Allemagne, une cinquantaine de récepteurs est distribuée parmi les fonctionnaires du Parti national-socialiste.

Étant donné cet accès limité au média, la rencontre entre la télévision et son public se fait presque exclusivement dans le cadre des expositions, limitant ainsi les potentialités du média à une vision à distance « de près ». À l'instar du pavillon de Telefunken installé en 1936 (Fig. 2), de nombreux dispositifs d'exposition offrent une vue sur un studio et sur l'image transmise par le récepteur placé devant ou à proximité de la vitrine. Au lieu de proposer une vision à distance, cette mise en scène

4. Karl Marx, Grundrisse, Heft V, janvier 1858.

5. Rudolph [sic] Arnheim, "Seeing Afar Off", Intercine, février 1935, n² 2, p. 77 : "We see the people gathered together in the central square of a near-by [sic] city, we see the head of the government of a neighbouring state, we see boxers fighting for the world's title on the other side of the ocean, we see an English jazz band, an Italian soprano, a German professor, the burning members of a train that has collided, the masked figures of carnival... We can admire the sun setting behind Vesuvius and a second later the illuminated night-signs of New York. The need for the descriptive word disappears as the barrier of foreign language vanishes. The world in all its vastness comes to our room. " 
exhibe à proximité l'infrastructure télévisuelle et la transmission d'une image d'un point $\mathrm{A}$ à un point $\mathrm{B}$. Pour des raisons techniques mais aussi scénographiques, la distance parcourue par le signal télévisuel est de quelques mètres seulement (Fig. 3).

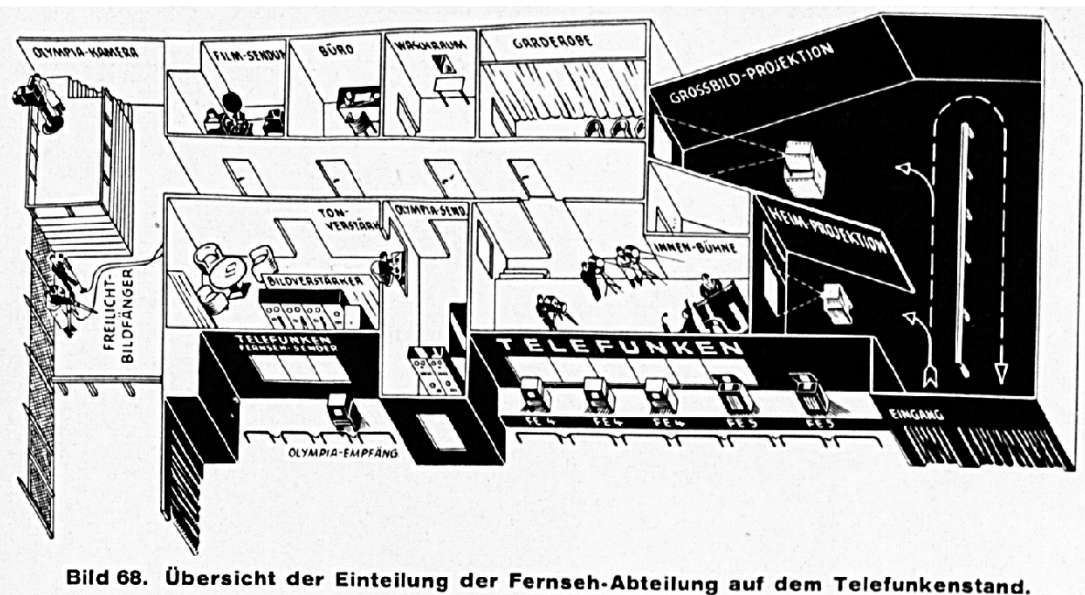

Fig. 2 : «Telefunken auf der 13. Grossen Deutschen Rundfunk-Ausstellung " (" Telefunken à la $13^{\mathrm{e}}$ Exposition allemande de radiophonie »), Telefunken-Zeitung, nº 74, 1936, p. 57.

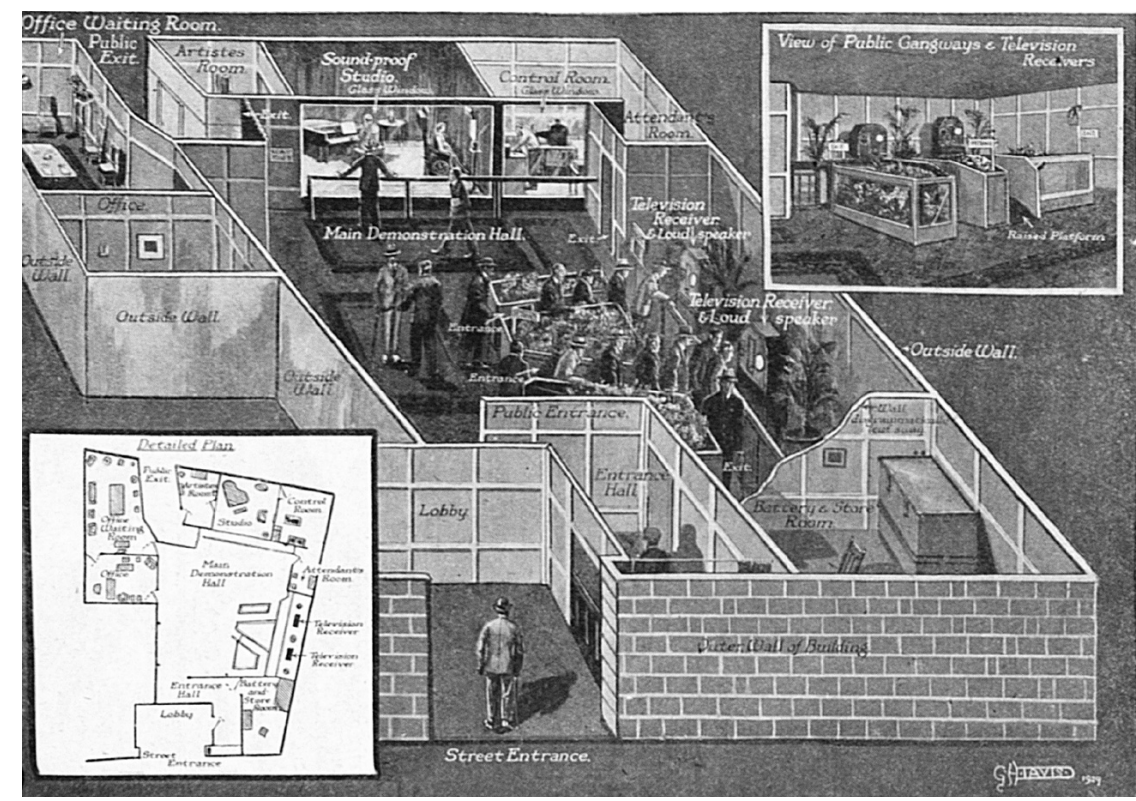

Fig. 3 : Exposition organisée par John Lodgie Baird, Londres, 1929. Leslie Trenton, « Now! Where do We Go from Here? » ("Et maintenant, vers où allons-nous ? "), Television $\mathrm{n}^{\circ} 3$, novembre 1929, p. 474. 
Juxtaposant la production de l'image et l'image produite, cette disposition architecturale des appareils permet en outre de faire expérimenter aux visiteurs la simultanéité télévisuelle. L'arrangement corrobore l'authenticité du direct télévisuel en dévoilant le réseau de machines et d'hommes nécessaires à sa production. Les paradigmes médiatiques de simultanéité, d'immédiateté ou d'ubiquité sont ainsi concrétisés au cœur d'une infrastructure technologique mise en scène dans les salles d'expositions.

Cette exhibition de la machinerie télévisuelle dicte un mode de réception particulier, avant tout articulé autour de l'attraction suscitée par l'appareil lui-même. Décrit presque exclusivement par des superlatifs, l'objet extraordinaire - et non pas son image transmise de loin - se retrouve au cœur des stands. La matérialité télévisuelle donnée en spectacle aux dépens du programme se reflète dans les modes d'adresse de l'appareil, destiné non pas à un téléspectateur, mais à un visiteur-promeneur mobile. Souvent, la taille très réduite des images nécessite un rapprochement de la part des visiteurs qui se penchent pour voir la transmission. Afin d'éviter que le public n'endommage des appareils fragiles et pour gérer la masse des curieux, des balustrades et autres outils scénographiques empêchent les visiteurs d'approcher de trop près des récepteurs ou contraignent le public à former une file afin d'examiner les machines individuellement (Fig. 4).

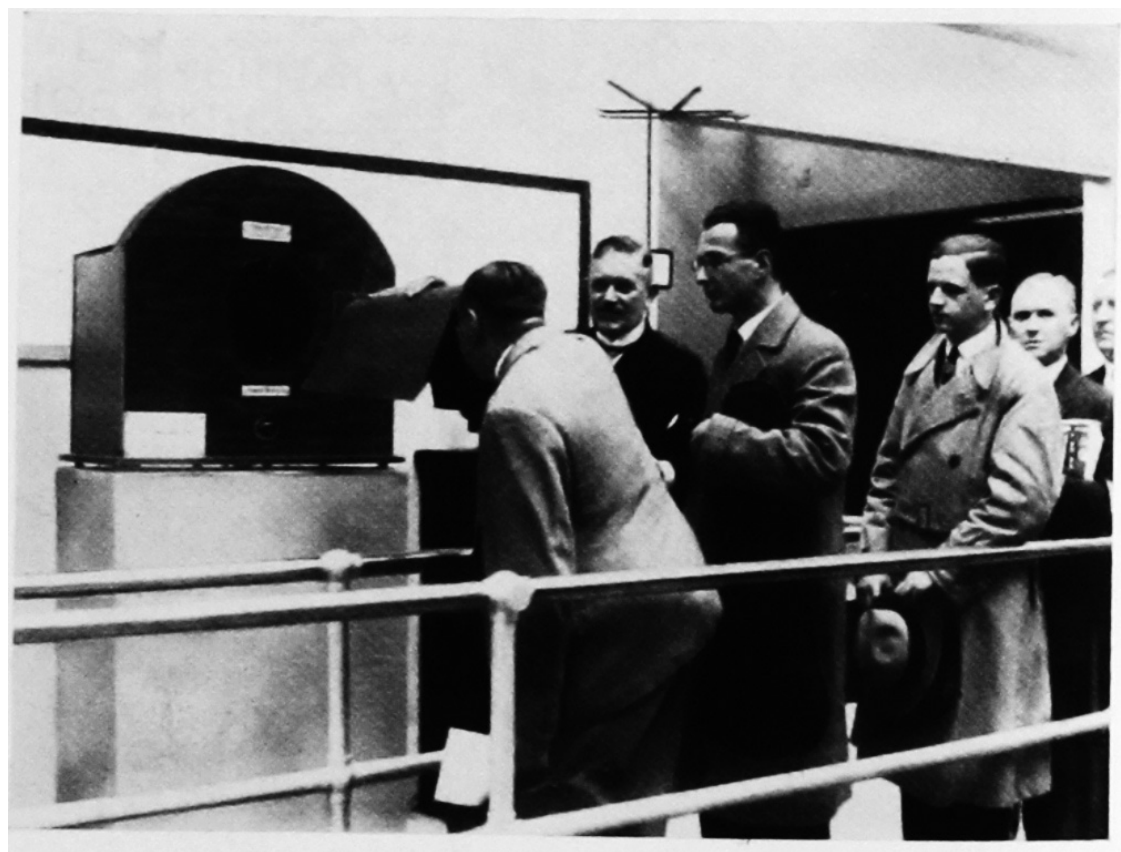

Fig. 4 : Exposition de radiophonie, Berlin 1928. Gerhart Goebel, «Das Fernsehen in Deutschland bis zum Jahre 1945 ", Archiv für das Post- und Fernmeldewesen, $\mathrm{n}^{\circ} 5$, août 1953 , p. 282 
AU SERVICE DE LA NATION : L'INAUGURATION DES SERVICES TÉLÉVISUELS DANS LES FOIRES

En rapprochant les appareils télévisuels et les spectateurs-visiteurs, l'espace d'exposition resserre l'échelle du média sur l'«ici et maintenant". À la place de conquêtes territoriales via la télévision, l'exposition limite la transmission à son propre espace. Cependant, malgré ces restrictions, l'objet télévisuel intègre le paysage médiatique national. La foire coconstruit le média comme preuve du progrès national et, tirant profit de son capital symbolique en tant que technologie à la pointe de la modernité, l'utilise pour célébrer les succès technologiques et scientifiques nationaux. Les foires de radiophonie à Berlin et à Londres, deux événements annuels organisés pour promouvoir l'industrie nationale, tout comme la New York World's Fair en 1939, jouent un rôle fondamental dans cette "nationalisation» du médium. À travers des scénographies modernisées mettant en valeur le nouveau média, les expositions traduisent l'ambition des responsables de lancer un service de télévision national.

\section{Communication et transport : construire l'image d'un régime moderne (Berlin, 1935)}

En Allemagne, des présentations publiques de la télévision sont organisées chaque année à partir de 1928 au sein de la Funkausstellung, la foire radiophonique de Berlin ${ }^{6}$. Réunie pour l'occasion, l'industrie allemande expose régulièrement les dernières innovations en matière de "vision à distance ", y compris des appareils pour la projection télévisuelle sur grand écran ou des systèmes de visiophonie. Si l'arrivée du régime nationalsocialiste en 1933 ne change pas immédiatement les pratiques d'exposition, ces dernières se voient radicalement transformées lors de la Funkausstellung 1935. Organisée en août, cette exposition suit l'annonce faite par la ReichsRundfunk-Gesellschaft en mars 1935 de l'ouverture d'un service public de télévision. Célébré comme «le premier programme télévisuel régulier du monde ${ }^{7}$ ", le service transmet de fait des extraits de films à un public très restreint et illustre ainsi le décalage existant entre les ambitions politiques et les réalités technologiques au milieu des années $1930^{8}$. Néanmoins, le démarrage du service télévisuel marque l'absorption du média dans la machinerie propagandiste du régime nazi et la Funkausstellung accorde dès lors une place centrale à la télévision. Pour la première fois en 1935 , le média est cité dans le slogan officiel de l'exposition, "Volkssender!

6. Pour une histoire de la Funkausstellung en général: Eva Susanne Bressler, Von der Experimentierbühne zum Propagandainstrument: die Geschichte der Funkausstellung von 1924 bis 1939, Cologne, Böhlau, 2009.

7. «Der erste Fernseh-Programmbetrieb der Welt ", Funk-Stunde, avril 1935, n 15, p. 564.

8. Klaus Winker, Fernsehen unterm Hakenkreuz: Organisation, Programm, Personal, Cologne, Böhlau, 1994, p. 70. 
Fernsehen! Volksempfänger ! " . Pour la première fois également, la salle dédiée à la télévision se distingue par une mise en scène homogène, la "Fernsehstrasse », la rue de la télévision ${ }^{10}$. Contrairement aux expositions précédentes où de nombreux appareils étaient simplement juxtaposés et où la scénographie soulignait surtout la multiplicité des dispositifs télévisuels développés (Fig. 5), la Fernsehstrasse propose un arrangement ordonné d'appareils (très) similaires (Fig. $6 \& 7$ ).
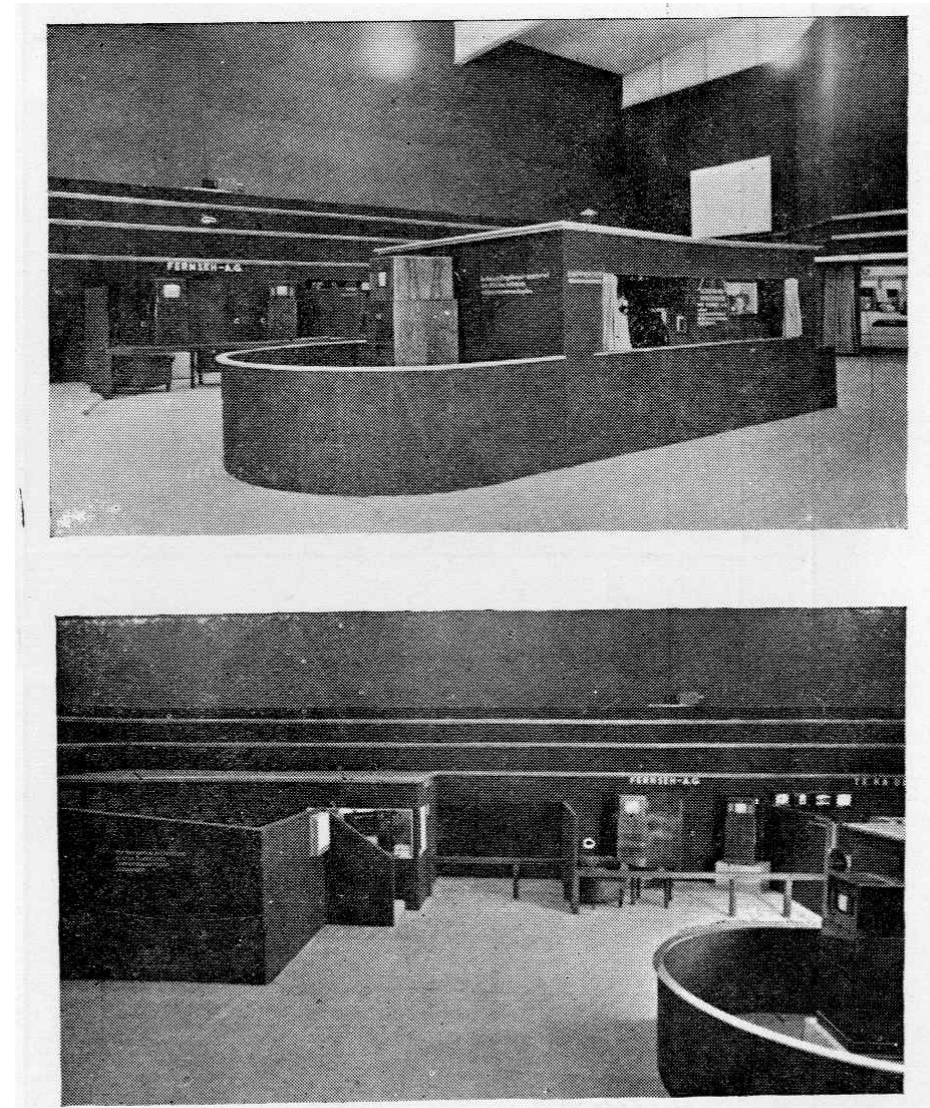

FIGS. 1 and 2-The Exhibition Hall.

Fig. 5 : Ernest H. Traub, « Television at the Berlin Radio Exhibition, 1934 »

( La télévision à l'Exposition radiophonique de Berlin, 1934 »), Journal of the Television Society 4, septembre 1934, p. 341.

9. Soit : «Émetteur du peuple ! Télévision ! Récepteur du peuple ! ».

10. À la différence des années précédentes, la salle de télévision est chapeautée par la Reichspost, la Reichsrundfunkgesellschaft et le ministère de l'Aviation. Georg Kette, "Fernsehen auf der Rundfunkausstellung 1935 ", Fernsehen und Tonfilm. Zeitschrift für Technik und Kultur des Fernsehwesens und des Tonfilms, $1935, \mathrm{n}^{\circ} 7$, p. 68. 


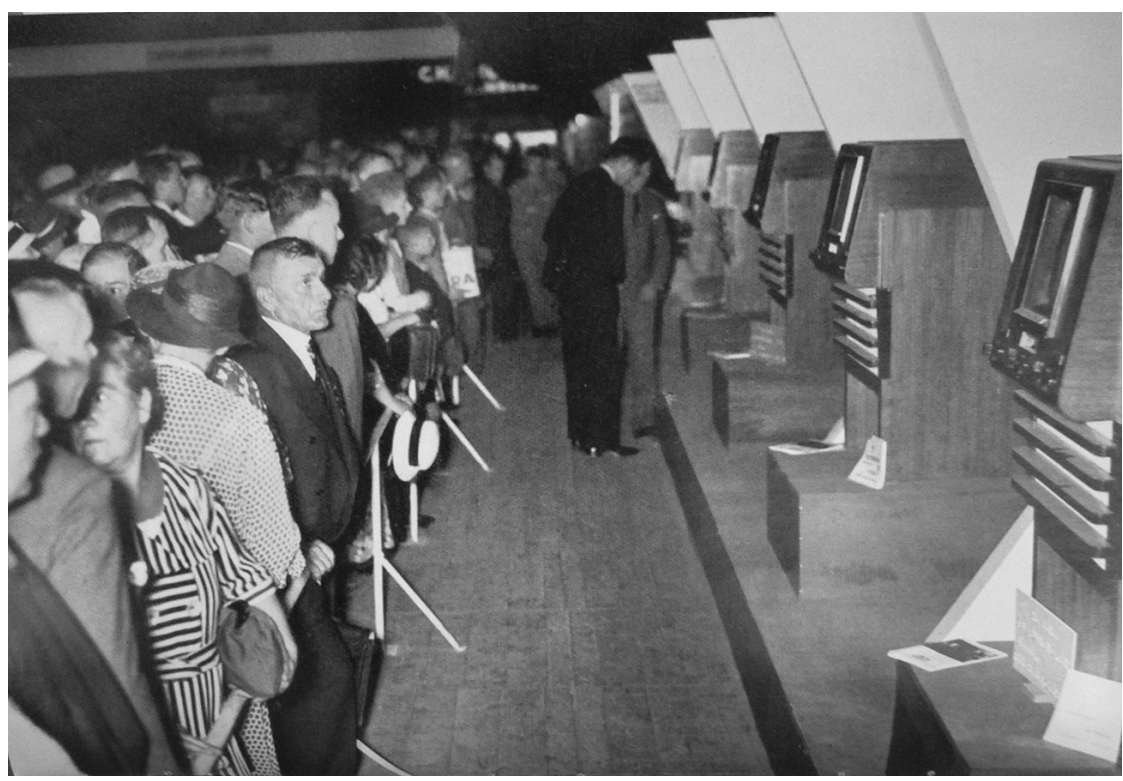

Fig. 6 : Amtlicher Führer zur 12. Grossen Deutschen Rundfunkausstellung Berlin 1936, Berlin, 1936, s. p. n.

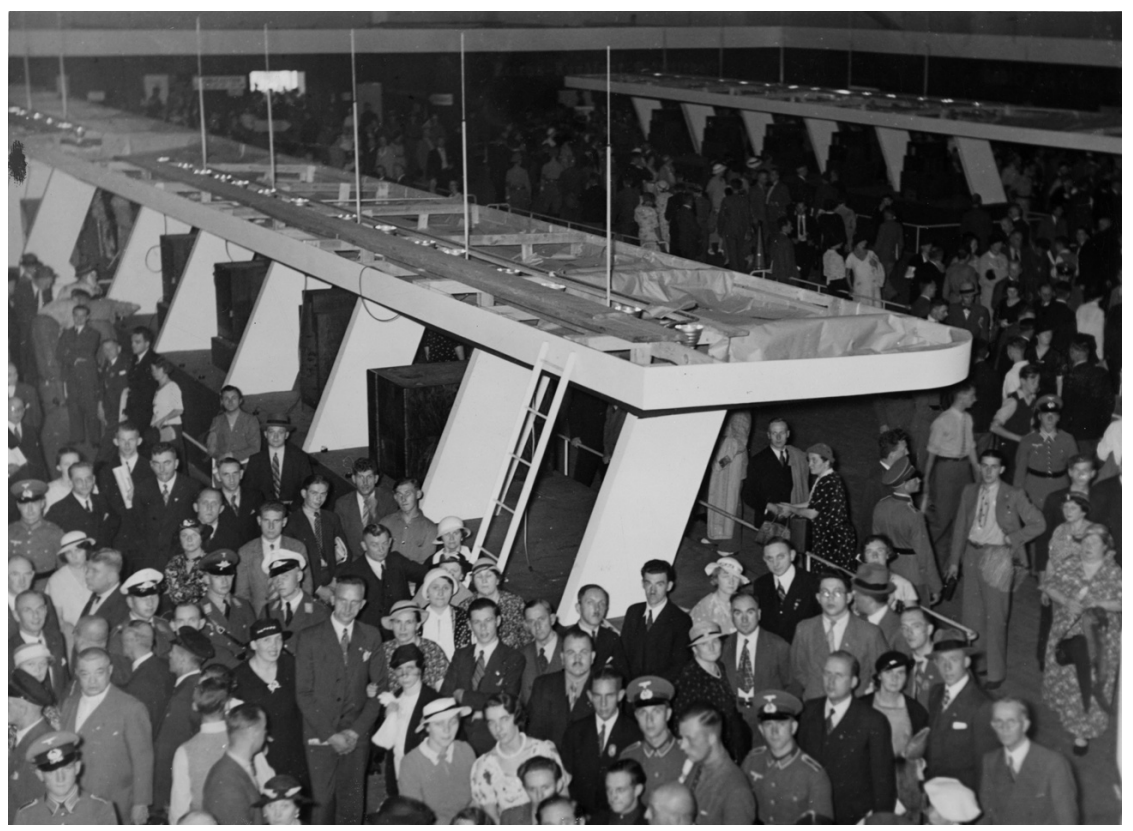

Fig. 7 : Fernsehstrasse (rue de la télévision), Funkausstellung 1935. Deutsches Rundfunkarchiv F030303-019. 
À travers cette disposition et cette mise en scène, la nouvelle scénographie renvoie au langage de la rationalisation et de la standardisation des processus de fabrication des appareils télévisuels : elle transforme des prototypes de laboratoire en des postes prétendument produits en masse. Alors même que le contenu audiovisuel demeure limité et que les récepteurs ne sont pas encore commercialisés, l'alignement de vingt postes de réception similaires sur deux rangées projette une image en trois dimensions d'un service télévisuel à la portée de tous. Cette disponibilité virtuelle de l'appareil télévisuel est renforcée par sa juxtaposition, dans le slogan de la foire, avec le Volksempfänger, le poste de radio fabriqué en série dès 1933. Conçu par le gouvernement pour être vendu à bas prix, le Volksempfänger promet de satisfaire les désirs consuméristes du peuple allemand tout en diffusant la voix du Führer dans chaque foyer ${ }^{11}$. Associant la télévision à cette icône médiatique du régime national-socialiste, l'exposition, et plus particulièrement la Fernsehstrasse, renvoient également à un autre emblème de la modernité allemande : la Volkswagen. À l'image du réseau des autoroutes nationales, la Volkswagen est censée élever la Volksgemeinschaft au rang des nations modernes: plus qu'un projet automobile, elle représente une entreprise idéologique et politique fournissant matière à l'autoreprésentation d'une nation supposément avancée ${ }^{12}$. À travers ces multiples références, la télévision exposée à la Funkausstellung est intégrée dans un dense réseau de signifiants qui renvoient à la modernité industrielle et à la production de masse et qui, ensemble, annoncent l'avènement de la société de consommation pour les membres de la communauté nationale-socialiste ${ }^{13}$.

\section{Redéfinir l'institution : la BBC en tant qu'entreprise audiovisuelle}

En Grande-Bretagne, l'ouverture officielle du service de la British Broadcasting Corporation (BBC), annoncée pour le 2 novembre 1936, est précédée par un programme expérimental transmis à Radiolympia, la foire radiophonique londonienne qui se déroule à la fin du mois d'août. Pour la première fois, le public londonien peut suivre les programmes de la BBC réalisés dans les studios d'Alexandra Palace, à quelques kilomètres des halls d'exposition. Contrairement aux éditions précédentes de Radiolympia,

11. Pour une analyse du Volksempfänger : Uta C. Schmidt, «Der Volksempfänger. Tabernakel moderner Massenkultur ", in Inge Marssolek et Adelheid von Saldern (dir.), Radiozeiten: Herrschaft, Alltag, Gesellschaft (1924-1960), Potsdam, Verlag für Berlin-Brandenburg, 1999, pp. 136-159. Également Wolfgang König, Volkswagen, Volksempfänger, Volksgemeinschaft. "Volksprodukte" im Dritten Reich: Vom Scheitern einer nationalsozialistischen Konsumgesellschaft, Paderborn, Schöningh, 2004.

12. Pour une analyse de la valeur symbolique de la Volkswagen: André Gunthert, "La voiture du peuple des seigneurs : naissance de la Volkswagen ", Vingtième Siècle. Revue d'histoire, juillet 1987, $\mathrm{n}^{\circ} 15$, pp. $29-42$.

13. Anne-Katrin Weber, «Du dispositif spectaculaire au poste standardisé : l'exposition de la télévision aux Funkausstellungen berlinoises, 1928-1939 " in Oliver Lugon (dir.), Exposition et médias : photographie, cinéma et télévision, Lausanne, L'Âge d'homme, 2012, pp. 271-293. 
d'où les démonstrations télévisuelles étaient bannies et où seuls quelques récepteurs étaient exposés sans transmettre de contenu, le nouveau média domine la foire en 1936. Omniprésente sur les stands des fabricants de postes dans le hall principal, la télévision est exposée avec les récepteurs de radio et s'insère ainsi dans la ronde des biens de consommation. De plus, un espace séparé comportant huit cabines de visionnage est réservé aux émissions télévisuelles. Chaque cabine accueille deux récepteurs dont le châssis est partiellement couvert par des rideaux dissimulant sa marque de fabrique. La promotion de l'appareil et la promotion du programme sont ainsi séparées dans l'espace : l'objet télévisuel est présenté dans le hall principal alors que l'image télévisuelle est montrée comme pure surface dans les cabines de démonstration. Introduisant une distinction entre les producteurs des postes - les fabricants - et la productrice du contenu télévisuel - la $\mathrm{BBC}$-, ce choix scénographique permet à cette dernière de s'affirmer comme unique responsable des programmes. Si l'industrie télécommunicationnelle s'occupe de la fabrication des téléviseurs, la BBC supervise désormais la création radiophonique et télévisuelle.

Cette redéfinition de la BBC en tant qu'entreprise audiovisuelle transparaît également à travers les multiples références à la vision, au regard et à l'image introduites dans l'exposition, à commencer par la mire précédant le programme expérimental montrant un œil, métaphore et organe de la (télé-)vision ${ }^{14}$. De même, un œil figure sur la couverture du premier numéro spécial de Radio Times, le journal de programme officiel de la $\mathrm{BBC}^{15}$. La chanson Here's Looking at You chantée par Helen McKay, un élément fixe des premières émissions à Radiolympia, fait également écho au motif de l'œil en décrivant la télévision comme un "seeing medium " (medium visuel) et contient une invitation adressée par la BBC à son public de «sit down and look" (s'asseoir et regarder) ${ }^{16}$.

Après le démarrage du service régulier, la démonstration télévisuelle à la foire radiophonique devient une attraction-phare permettant à la BBC d'asseoir davantage son mandat télévisuel. En 1938, la BBC vend un « livret illustré " ("pictorial booklet») intitulé And Now. The British Broadcasting Corporation Presents Television To The World ${ }^{17}$. Composée presque exclusivement de photographies, la brochure présente le passé et l'actualité de la télévision à la BBC. À Radiolympia, ce récit en images est complété par un studio vitré invitant les visiteurs à assister à la production d'une

14. "First Television Broadcast " The Times, 26 août 1936.

15. Pour les archives du magazine mises à disposition par la BBC: http://www.bbc.co.uk/ historyofthebbc/research/general/radio-times/pre-war.

16. La chanson peut être écoutée sur http://www.youtube.com/watch?v $=$ pqDgk $4 \mathrm{NcrCw}$ : "Here's Looking at You / From out of the Blue / Don't make a fuss / But settle down and look at us: / Here's looking at you / it seems hardly true / That radio can let you sit and watch a show / This wonderful age goes to show / That all the world's a stage, / First you heard, now you see, / As you wonder what the next thing / On the list will be, / What hullabaloo! / We're just peeping through /To say 'how do' / Here's Looking at You ".

17. BBC Archives, TV Publicity Pamphlet 1938, T 23 / 80, "Radiolympia Report ", The Wireless and Electrical Trader, $1938, \mathrm{n}^{\circ} 732$, p. 374. 
émission télévisuelle (Fig. 8) ${ }^{18}$. En montrant " exactement comment les spectacles sont réalisés dans le studio d'Alexandra Palace ", il exhibe le travail habituellement caché des cameramen et des producteurs, des décorateurs et autres employés de la $\mathrm{BBC}^{19}$. La transparence assumée par l'institution permet de fidéliser son public à qui elle donne littéralement tout à voir, et, partant, de consolider davantage le récit de son évolution, d'une institution radiophonique à une entreprise audiovisuelle.

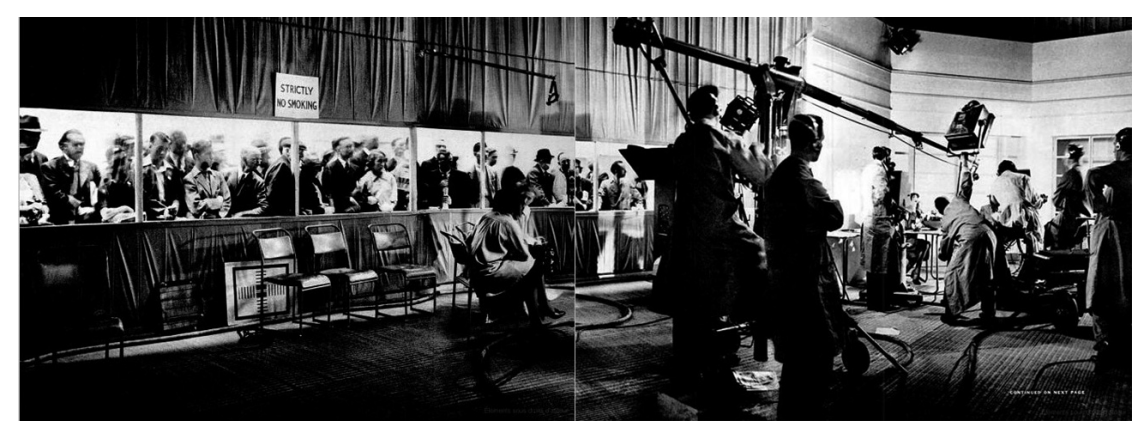

Fig. 8: "Television. It Makes Its Commercial Debut This Spring With World's Fair » ("La télévision: Elle fait ses débuts commerciaux ce printemps à la Foire mondiale "), Life, $\mathrm{n}^{\circ} 8,20$ février 1939 , p. $45^{20}$.

Lors de la dernière édition de Radiolympia avant la guerre, l'intégration de la télévision au sein de la BBC - et donc dans le paysage médiatique national - est explicitement mise en scène dans le hall principal. Deux grandes maquettes de la Broadcasting House et d'Alexandra Palace, soit respectivement des studios de radio et de télévision, sont construites en vis-à-vis ${ }^{21}$. Surplombant l'espace où l'industrie expose ses produits télécommunicationnels, ils matérialisent la double responsabilité de l'institution radiophonique et télévisuelle : si les deux médias sont désormais constitutifs de la $\mathrm{BBC}$, cette dernière est indispensable au paysage médiatique britannique dans son ensemble.

Commercialiser la télévision pour tout le pays : le média à la New York World's Fair

Aux États-Unis, la Radio Corporation of America (RCA) profite de l'occasion unique offerte par la New York World's Fair à la fin des

18. "News of the Week: Radiolympia. Television the Focal Point of the Show ", Wireless World and Radio Review, n 991, 25 août 1938, p. 187.

19. "Wait for Radiolympia! ", Radio Times, 22 juillet 1938, p. 16 : "exactly how shows are produced in the studio at Alexandra Palace".

20. Cet article du magazine américain Life examine l'état de la télévision à la fin des années 1930 en vue des démonstrations que la RCA s'apprête à réaliser dans le cadre de la New York World's Fair (voir ci-dessous) ; il utilise cette photographie de Radiolympia pour illustrer les progrès réalisés par la BBC.

21. Brochure publicitaire destinée aux exposants. Archive Earls Court, Londres. 
années 1930 pour mettre en scène la «naissance d'une industrie ${ }^{22}$ ». À travers sa compagnie de radiodiffusion, la National Broadcasting Company (NBC), la RCA lance le service télévisuel en transmettant en direct le discours d'inauguration de la foire du président Roosevelt. La mise en scène spectaculaire de la RCA est le fruit d'une action stratégique planifiée de longue date qui lui permet de promouvoir ses recherches sur la télévision auprès d'un large public et de se positionner comme leader dans un champ émergent face aux entreprises concurrentes. Sa présence à la World's Fair est en outre censée inciter la Federal Communication Commission (FCC) à approuver le service télévisuel commercial à un moment où cette dernière enquête sur les stratégies monopolistiques des grandes entreprises de télécommunication ${ }^{23}$.

Dès lors, il n'est pas surprenant de constater que la RCA multiple les écrans et les appareils télévisuels dans son pavillon. Dans le "Hall of Television", treize postes transmettent le programme de la NBC; dans la salle principale du pavillon sont exposés un récepteur transparent ainsi qu'un modèle de laboratoire. Un autre téléviseur est intégré au "Radio Living Room of Tomorrow» (le "Salon radio de demain »), une salle de séjour futuriste équipée également d'une radio, un phonographe, un récepteur de fac-similé et un projecteur pour les "home movies ${ }^{24}$ ".

La télévision est également au cœur des grandes peintures murales réalisées par Louis Ferstadt. Quatre grands tableaux intitulés Broadcasting, Manufacturing, Research et Communication occupent les trois-quarts des murs du hall principal et guident le visiteur à travers les différents thèmes de l'exposition (fig. 9). Chaque peinture murale combine des figures abstraites et des dessins figuratifs, tandis que des objets complémentaires sont placés à côté ou sous les tableaux muraux. Dominant l'intérieur du pavillon, les peintures présentent les multiples domaines d'activité de la RCA qui regroupe notamment une compagnie de radioet de télédiffusion, des laboratoires de recherche et un secteur de manufacture. À travers elles, l'hétérogénéité et la diversité de l'entreprise sont transformées en un ensemble harmonieux et esthétiquement valorisé. Célébrant la RCA, les tableaux font également office de publicité pour toute l'exposition. Grâce à leurs "motifs colorés " (" colorful pattern of forms "), les tableaux muraux sont "suffisamment grands et frappants

22. David Sarnoff, "The Birth of an Industry ", in David Sarnoff, Pioneering in Television, Prophecy and Fulfillment: Excerpts from Speeches and Statements, New York, Dept. of Information, RCA, 1948, pp. $40-41$.

23. Ron Becker, "Hear-and-See Radio" in the World of Tomorrow: RCA and the Presentation of Television at the World's Fair, 1939-1940", Historical Journal of Film, Radio E Television, octobre 2001, $\mathrm{n}^{\circ}$ 4, pp. 361-378. Également: Andreas Fickers, "Presenting the "Window on the World" to the World. Competing Narratives of the Presentation of Television at the World's Fairs in Paris (1937) and New York (1939) ", Historical Journal of Film, Radio E Television, $\mathrm{n}^{\circ}$ 3, août 2008, pp. 291-310.

24. George H. Clark Radioana Collection, Archives Center, National Museum of American History, Smithsonian Institution, série 112, box 311, George H. Clark, "RCA at the Fair ». 
pour être facilement compris à distance ${ }^{25}$ »; ils peuvent aussi être vus par les passants depuis l'extérieur à travers l'immense baie vitrée du pavillon. Ainsi, le public accède-t-il à la carte de visite peinte de la RCA sans même entrer dans l'exposition. Finalement, l'art mural monumental se substitue à la visualité réduite du média qu'il est censé promouvoir. Le surplus de visibilité des peintures qui entourent le visiteur contrebalance la surface réduite des écrans télévisuels; le "nouveau média " est remplacé par une "ancienne" technique. De la sorte, la peinture murale transcende l'exclusivisme que la télévision doit à son prix élevé. Promu par le New Deal, le mur peint est devenu l'art le plus démocratique des années 1930 : adopté par la RCA, il confêre à ses produits commerciaux une image d'accessibilité à tous ${ }^{26}$.

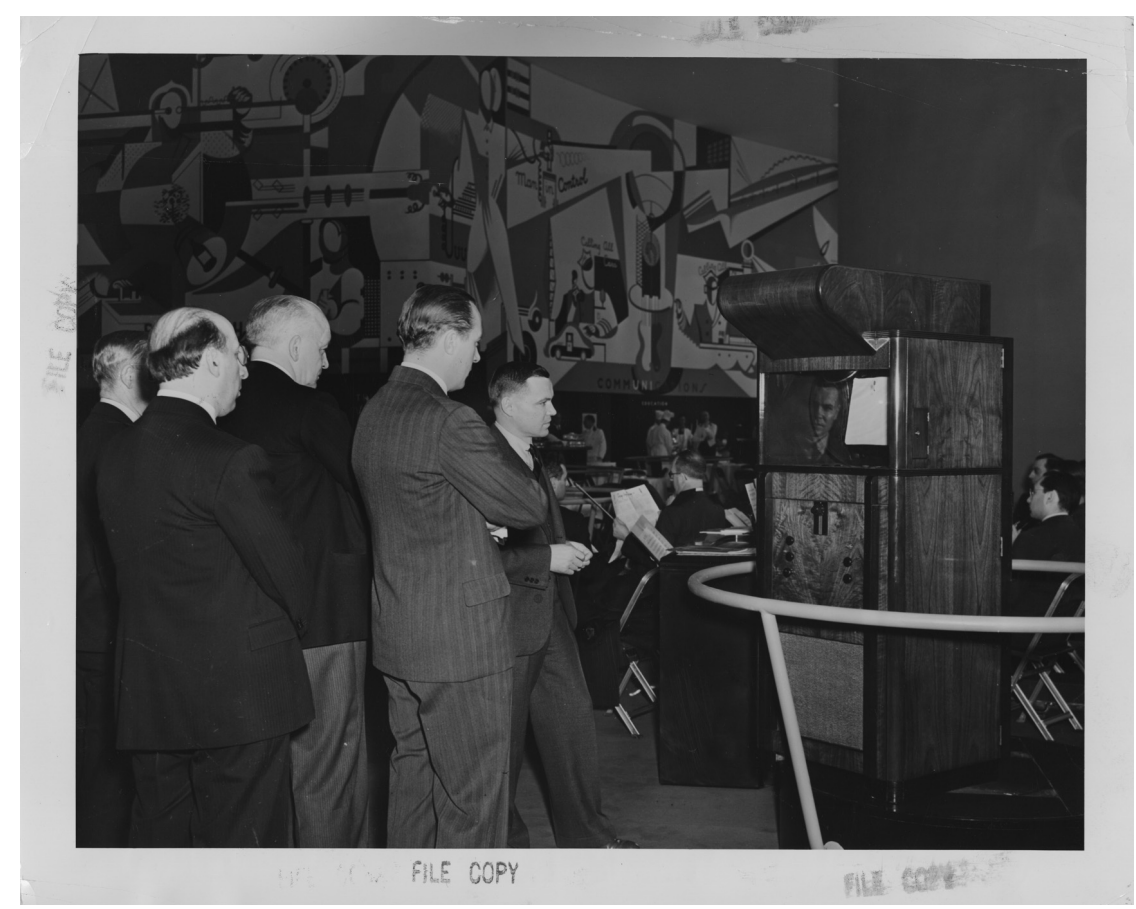

Fig. 9 : Manuscripts and Archives Division, The New York Public Library.

"Radio Corporation of America (RCA) - Group at dedication inspecting television ", The New York Public Library Digital Collections. 1935-1945.

http://digitalcollections.nypl.org/items/5e66b3e8-f864-d471-e040-e00a180654d7

25. Ibid., p. 3 : "strong and large enough to be understood clearly from a distance".

26. Jody Patterson, " Modernism and Murals at the 1939 New York World's Fair », American Art, $2010, n^{\circ} 2$, pp. 50-73. 
En Allemagne, en Grande-Bretagne et aux États-Unis, le service public télévisuel est inauguré en lien étroit avec l'exposition. Servant de tremplin au nouveau média, l'espace de l'exposition participe à la consolidation de l'organisation nationale des institutions télévisuelles et assoit la place du média au sein de chaque paysage médiatique, qui repose respectivement sur le contrôle étatique, le service public centralisé et l'organisation commerciale. En Allemagne, la Funkausstellung élève la télévision au rang d'un média de masse et, en l'associant à d'autres projets techno-idéologiques le Volksempfänger et la Volkswagen -, contribue à fonder l'imaginaire de la société de consommation nationale-socialiste. À Londres, la foire de 1936 précède l'ouverture officielle du service régulier et sert de ballon d'essai à la $\mathrm{BBC}$, institution radiophonique qui cherche à se repositionner comme institution audiovisuelle. Aux États-Unis, le pavillon de la RCA à la New York World's Fair est l'expression de l'organisation privatisée des médias et présente la compagnie comme étant la seule responsable de la télévision américaine.

Au-delà des particularités nationales, la présence de la télévision au sein de l'espace de l'exposition est néanmoins déterminée, au milieu des années 1930, par le contexte international. Fréquentées par la presse nationale et étrangère, les expositions sont documentées bien au-delà de la seule villehôte. Les photographies des stands les plus attractifs circulent internationalement et contribuent à la diffusion suprarégionale ou supranationale de la télévision. Les visites des journalistes dans des foires étrangères facilitent l'évaluation des différences nationales en matière technologique et institutionnelle et permettent de définir le modèle national par opposition avec les institutions étrangères ${ }^{27}$. Dans l'entre-deux-guerres, la foire représente ainsi un nœud au cœur d'un réseau d'échanges transnationaux qui façonne la télévision.

En effet, l'ouverture des services publics au milieu des années 1930 et leur mise en scène respective au sein des foires ne peuvent se comprendre en dehors du contexte de la compétition technologique, industrielle et politique internationale. Selon l'historien Klaus Winker, auteur d'une importante étude sur la télévision "sous la Swastika ", le service lancé par l'Allemagne traduit de prime abord «l'ambition politique du régime nazi à vouloir devancer la $\mathrm{BBC}$ anglaise et toutes les autres nations

27. Les journaux envoient régulièrement leurs propres journalistes à des foires à l'étranger et/ou invitent des journalistes étrangers à écrire sur la foire nationale. Par exemple, "Impressions of the Berlin Show ", The Wireless World, 12 septembre 1928, et Alfred Gradenwitz, "Television at the Berlin Radio Exhibition ", Television, 1930, n 32, pp. 327-328. Concernant l'importance du modèle américain pour la définition du service télévisuel de la BBC et vice versa : Michele Hilmes, Network Nations: A Transnational History of British and American Broadcasting, New York, Routledge, 2011. 
grâce à cette inauguration hâtive ${ }^{28}$. Plus précisément, le lancement du service berlinois en mars 1935 est une réaction à la constitution du Selsdon Committee en Grande-Bretagne, un groupe d'experts créé en 1934 et mandaté pour débattre de l'avenir de la télévision anglaise. Durant plusieurs mois le comité rencontre de nombreux acteurs de l'industrie des télécommunications et d'autres organismes intéressés et investigue les différentes questions liées à l'ouverture d'un service public ${ }^{29}$. Le mandat du Selsdon Committee comporte également des voyages aux États-Unis et en Allemagne où les membres visitent notamment le département de recherche de la Reichsrundfunkgesellschaft et plusieurs entreprises pri$v^{2} e^{30}$. Révélant les ambitions britanniques, ces investigations non seulement mènent au lancement du service public anglais, mais stimulent la réorganisation du paysage médiatique en Allemagne ${ }^{31}$.

On observe également ces rivalités européennes aux États-Unis où la presse annonce en 1935 le début d'une "ruée télévisuelle mondiale » et une "course télévisuelle» internationale ${ }^{32}$. «La Grande-Bretagne est prête à offrir un service public de Radio-Télévision "; "L'entrée de la GrandeBretagne dans la course à la télévision secoue l'Amérique »; "La Télévision démarre pour les Allemands dans une semaine "; "Course à la télévision. L'Angleterre trouve que la compétition est féroce "; "Expositions européennes: pas de télévision à l'exposition radiophonique de Londres - Berlin a montré des récepteurs d'images $n^{33}$ : ces titres d'articles traduisent le capital symbolique de la télévision comme témoin du progrès d'une nation et confirment l'importance du contexte transnational pour le projet de télévision nationale.

$\mathrm{Au}$ sein des expositions, des dispositifs scénographiques similaires, voire identiques, confirment la circulation entre les personnes, les objets et les idées, et témoignent de la capacité de la télévision à s'adapter à des contextes nationaux et idéologiques très différents. Ainsi, la télévision transparente exposée au centre du pavillon de la RCA en constitue-telle une attraction phare, mais certes pas unique (Fig. 10) : en 1936, à Londres, la marque Cossor expose un modèle similaire ; en 1937, la firme allemande

28. Klaus Winker, op. cit., p. 71 : "machtpolitischer Ehrgeiz des NS-Regimes, mit dem hastigen Start der englischen BBC und allen anderen Nationen zuvorkommen zu wollen ".

29. Pour une histoire détaillée du Selsdon Committee : R.W. Burns, British Television: The Formative Years, IET, 1986, pp. 302-359.

30. "The Television Committee in Germany ", Television, décembre 1934, p. 538.

31. William Uricchio, "Introduction to the History of German Television, 1935-1944", Historical Journal of Film, Radio and Television, 1990, n 2, p. 175.

32. "World television rush is On! ", Radio World, 16 février 1935, n²3, p. 4 ; "Television Race Gets Hotter ", Radio World, 17 septembre 1935, nº 8, pp. 5-6.

33. "Britain is Ready to Give Public Radio Television ", Chicago Daily Tribune, $1^{\mathrm{er}}$ février 1935 ; "England's Entry in Television Race Stirs America ", The New York Times, 10 février 1935 ; "Television For Germans Will Start in Week", Chicago Daily Tribune, 9 mars 1935 ; "Television Race. England finds competition is keen ", The New York Times 30 juin 1935 ; "European Exhibits: No Television Seen At London's Radio Show - Berlin Displayed Picture Receivers ", The New York Times, 8 septembre 1935 . 
Telefunken présente un récepteur comparable ${ }^{34}$. Évoquant les horizons illimités promis par l'imaginaire télévisuel décrit au début de cet article, l'appareil mis à nu renvoie à une définition médiatique internationale de la « vision à distance ». Plus surprenant, la " Fernsehstrasse » voyage en 1939 de Berlin à Londres où un dispositif scénographique similaire est montré à Radiolympia. Installée sur le balcon entourant le hall principal, la «Television Avenue » anglaise est constituée de soixante-quatre récepteurs, dont la présence est annoncée sur l'affiche de la foire ${ }^{35}$.

Ces observations ne cherchent pas à nier les différences engendrées par les contextes démocratiques et autoritaires dans l'histoire de la télévision, mais elles permettent de souligner certaines similitudes. Étudiées séparément, les foires britanniques, américaines et allemandes apparaîtraient toutes comme des cas particuliers : dans une perspective comparative, elles révèlent au contraire de nombreuses homologies qui nous renseignent aussi bien sur l'avènement de la télévision que sur l'histoire des expositions elles-mêmes.

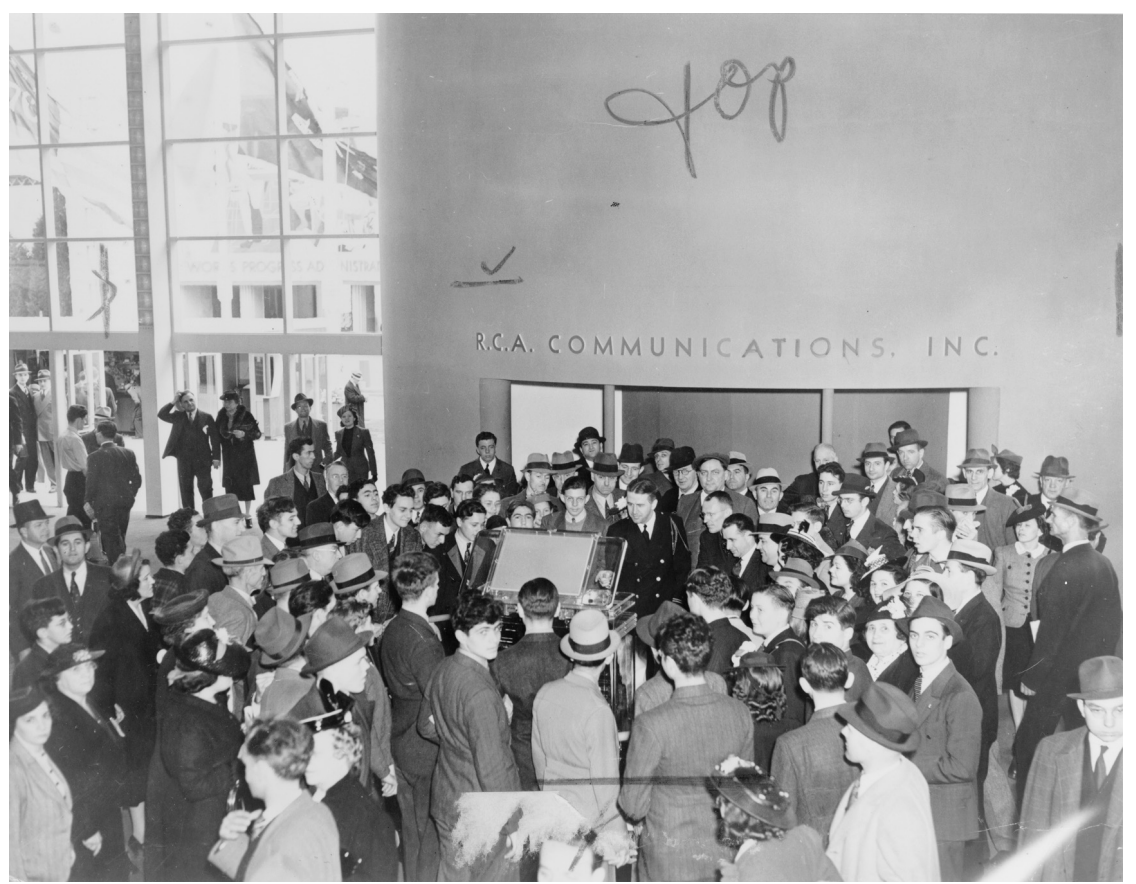

Fig. 10 : «La foule entoure la télévision à l'exposition de la RCA à la World's Fair de 1939 », 1939, New York World-Telegram and the Sun Newspaper Photograph Collection, Library of Congress, LC-USZ62-116255.

34. Une image du récepteur Cossor est disponible sur http://www.scienceandsociety.co.uk/ results.asp? image $=10307874$.

35. Affiche Radiolympia, Wireless World, 24 août 1939, p. 7. Également : "This Month's great show ", The Observer, 13 août 1939. 
CONCLUSION

La tension entre l'ubiquité imaginaire de la télévision et sa définition nationale que décrit cet article traverse l'histoire du média. Ainsi, après la Deuxième Guerre mondiale, la dimension utopique de la télévision conçue comme outil au service des échanges entre les peuples trouvet-elle une expression institutionnelle dans l'Union européenne de la radiodiffusion, l'UER. Fondée en 1950, l'Union est pensée par ses promoteurs comme une organisation au service d'un idéal européen et de la compréhension mutuelle entre les États ; à partir de 1953, elle soutient activement l'échange de programmes entre ses membres ${ }^{36}$. Malgré ses ambitions transnationales, l'UER contribue cependant de manière limitée à la création d'un espace socio-culturel étendu. L'échange de programmes au service d'une compréhension internationale débouche souvent sur une appropriation nationale ${ }^{37}$. Plus généralement, lors de son institutionnalisation durant les années 1950 en Europe et aux États-Unis - alors qu'elle devient enfin cette "fenêtre sur le monde " tant attendue - la télévision transmet tout d'abord des images de la et pour la "communauté imaginaire " nationale. Intégrée dans les organismes nationaux de communication, et en particulier dans les structures existantes de la radiophonie, elle sert d'expression et de témoin à l'identité nationale ${ }^{38}$.

Dans l'entre-deux-guerres, ni les infrastructures ni les programmes télévisuels ne contribuent à la consolidation de l'identité nationale et ils n'assument pas d'échanges de programmes transnationaux. Confinée aux sites d'exposition, la télévision n'offre que très peu de contenu médiatique. Face à l'absence de ce qui constituerait le "véritable " média, l'historiographie de la télévision des années 1920 et 1930 s'est concentrée principalement sur l'évolution technique, souvent racontée à travers les biographies des premiers inventeurs. L'analyse de la télévision exposée révèle l'enchevêtrement des espaces régionaux et transnationaux au centre desquels se situe la télévision et éclaire l'importance des foires pour la construction symbolique de celle-ci en tant que média national.

Anne-Katrin WeBer

Université de Lausanne

36. Patrick Alves, "L'Union européenne de radiodiffusion, 1950-1969 ", Bulletin de l'Institut Pierre Renouvin, 2007, n² 2, p. 23.

37. Christian Henrich-Franke, "Creating Transnationality Through an International Organization? ", Media History, 2010, n 1, p. 79. François Vallotton, "La Société suisse de radiodiffusion et télévision: coproduction et échange de programmes télévisés (1950-1970), " in Marie-Françoise Lévy et Marie-Noële Sicard (dir.), Les Lucarnes de l'Europe: Télévisions, cultures, identités 1945-2005, Paris, Publications de la Sorbonne, 2008, pp. 71-85.

38. Andreas Fickers, « National Barriers for an Imag(e)ined European Community: The TechnoPolitical Frames of Postwar Television Development in Europe ", Northern Lights. Film and Media Studies Yearbook, 2006, vol. IV, pp. 15-36 ; Sonja de Leeuw et al., "TV Nations or Global Medium? European Television Betweeen National Institution and Window on the World, » in Jonathan Bignell and Andreas Fickers (dir.), A European Television History, Malden, MA ; Oxford, Wiley-Blackwell, 2008, pp. 127-153. 